\title{
DETECCIÓN DE TUBERCULOSIS EN EL SERVICIO DE EMERGENCIA: UTILIDAD DE LA SEGUNDA BACILOSCOPÍA REALIZADA EL MISMO DÍA
}

\author{
Gina Tello ${ }^{1, a}$, Martha Ugarte ${ }^{1, b}$, Juan Agapito ${ }^{1,2, c}$, Alonso Soto ${ }^{3, d}$
}

\begin{abstract}
RESUMEN
La realización de una baciloscopía el mismo día de la atención del paciente es una estrategia que recientemente ha sido adoptada por la Organización Mundial de la Salud. Nuestro estudio buscó determinar el rendimiento diagnóstico adicional de una segunda baciloscopía tomada en un mismo día en pacientes con síntomas respiratorios, atendidos en el servicio de emergencia de dos hospitales de Lima. Se incluyeron 270 pacientes desde enero a noviembre de 2011, a los cuales se les solicitó dos muestras de esputo con un lapso de dos horas; las muestras fueron procesadas mediante el método de ZiehI Neelsen. La frecuencia de baciloscopías positivas fue del 18,5\%. El rendimiento diagnóstico adicional de la segunda baciloscopía fue 20,9\%. Considerando la alta frecuencia de tuberculosis diagnosticada por baciloscopía, se sugiere que esta prueba sea incluida de manera rutinaria en las emergencias de los hospitales de Lima Metropolitana.
\end{abstract}

Palabras clave: Tuberculosis; Esputo; Signos y síntomas respiratorios; Perú (Fuente: DeCS BIREME)

\section{TUBERCULOSIS DETECTION IN THE EMERGENCY ROOM: UTILITY OF SAME DAY SMEARS}

\begin{abstract}
Conducting a same day smears is a strategy that has recently been adopted by the World Health Organization. Our study sought to determine the additional diagnostic yield of a second sputum smear taken on the same day among patients with respiratory symptoms, attended at the emergency room from two hospitals in Lima. We included 270 patients from January to November 2011, which were asked two samples of sputum within two hours; the samples were processed by the Ziehl Neelsen method. The frequency of positive sputum smears was $18.5 \%$. The additional diagnostic yield for the second smear was $20.9 \%$. Considering the high prevalence of tuberculosis diagnosed by sputum smear, it is suggested that this test be included routinely in the emergency rooms of hospitals in Lima Metro area.
\end{abstract}

Key words: Tuberculosis; Sputum; Signs and symptoms, respiratory; Peru. (Source: MeSH NLM)

\section{INTRODUCCIÓN}

La tuberculosis pulmonar es una enfermedad frecuente en los servicios médicos y los servicios de emergencia. Los servicios de emergencia atienden en condiciones de hacinamiento a una gran cantidad de pacientes, lo que puede favorecer la transmisión de bacilos hacia otros pacientes, y al personal de salud.

El riesgo de adquirir tuberculosis varía según el tipo de instalación; el tiempo de permanencia en el nosocomio; la prevalencia de tuberculosis en la comunidad; el grupo ocupacional de los trabajadores; el área de la instalación en que trabajan; y la efectividad del control (1-4). Debido a que el riesgo de adquirir tuberculosis en los hospitales es 2 a 50 veces más que en la comunidad, el profesional de salud encargado de áreas críticas debe estar atento a la posibilidad de que aquellos pacientes que requieren atención de emergencia tengan tuberculosis, no solo para considerar dicho diagnóstico en la aproximación clínica sino para evitar la diseminación de la enfermedad.

Es por ello que la detección de casos de tuberculosis y el aislamiento respiratorio deben ser consideradas actividades prioritarias en la atención de pacientes en emergencia en países con alta prevalencia de

Escuela de Tecnología Médica, Facultad de Medicina, Universidad Peruana Cayetano Heredia. Lima, Perú

Instituto de Medicina Tropical Alexander von Humboldt, Universidad Peruana Cayetano Heredia. Lima, Perú

Hospital Nacional Hipólito Unanue. Lima, Perú

a Tecnólogo médico en Laboratorio Clínico; ${ }^{\mathrm{b}}$ tecnólogo médico en Urgencias Médicas y Desastres; ${ }^{\mathrm{c}}$ microbiólogo; ${ }^{\mathrm{d}}$ médico internista magíster en Estadística Aplicada

* Este trabajo se basa en la tesis para obtener el grado de bachiller: "Frecuencia de baciloscopías positivas en pacientes con afecciones respiratorias en la unidad de emergencia de dos hospitales de Lima”, realizada por GT y MV el 2012. 
tuberculosis, como el Perú. Pese a ello, es común el retraso o la omisión en la solicitud de baciloscopías en emergencia, y son pocos los estudios que han abordado este problema.

En un estudio realizado el año 2008, en la unidad de emergencia del Hospital Cayetano Heredia, de Lima, se encontró que de 3122 pacientes atendidos, 487 presentaron síntomas sugerentes de tuberculosis pulmonar; $31 \%$ tuvieron cultivo positivo y, de estos, $78 \%$ tuvieron baciloscopía positiva (5); ello evidenció que existe una frecuencia considerable de pacientes que acuden a la emergencia y que presentan síntomas sugestivos de tuberculosis pulmonar.

Dentro de las estrategias dirigidas a disminuir el retraso en el diagnóstico de tuberculosis pulmonar está la baciloscopía del mismo día (Frontloaded o Same day sputum microscopy) ${ }^{(6)}$, que consiste en la obtención de dos o más muestras durante el mismo día de atención del paciente, en contraposición a la solicitud convencional de realizarla en días sucesivos.

Existen estudios en población ambulatoria que han demostrado la utilidad de esta estrategia, indicando su equivalencia en cuanto a rendimiento diagnóstico, con la estrategia convencional de días sucesivos ${ }^{(7,8)}$. La baciloscopía del mismo día forma parte de las recomendaciones de la Organización Mundial de la Salud para la mejora en el diagnóstico de la tuberculosis ${ }^{(6)}$.

A pesar de ello, no existen estudios nacionales que hayan evaluado la factibilidad y utilidad de esta estrategia. Además, a pesar que se ha sugerido que existe una alta prevalencia de tuberculosis en servicios de emergencia, son pocos los estudios que han evaluado estrategias específicas para abordar dicho problema. En este contexto, nuestro estudio tuvo por objetivo evaluar el rendimiento diagnóstico adicional de una segunda baciloscopía realizada el mismo día, en pacientes que acuden por emergencia en dos hospitales de Lima, Perú. Además, se evaluó las características asociadas a presentar una baciloscopía positiva.

\section{EL ESTUDIO}

La población de estudio estuvo conformada por pacientes mayores de 14 años que acudieron al Servicio de Emergencia del Hospital Nacional Cayetano Heredia $(\mathrm{HNCH})$ en el periodo de enero a julio de 2011, y al Hospital Nacional Hipólito Unanue (HNHU) de agosto a noviembre de 2011; dichos pacientes presentaron algún síntoma respiratorio incluyendo tos, expectoración, disnea o hemoptisis; independientemente del tiempo que lo presenten; y que se encuentre en el servicio de emergencia por al menos seis horas. Se excluyeron aquellos pacientes con diagnóstico de tuberculosis en tratamiento y con incapacidad para expectorar.

El tamaño de muestra se calculó en base a una frecuencia estimada de baciloscopías positivas del $20 \%{ }^{(5)}$, una precisión de $\pm 5 \%$ y un rendimiento diagnostico adicional del 10\%; por lo que se previó tomar 240 pares de muestras.

Las muestras recolectadas en ambos hospitales fueron procesadas en el Laboratorio de Mycobacterias del $\mathrm{HNCH}$. En el procesamiento de las muestras, estas fueron decontaminadas con $\mathrm{NaOH}$ al $4 \%$; posteriormente, se realizó la coloración utilizando el método de Ziehl Neelsen, de acuerdo con las guías nacionales ${ }^{(9,10)}$.

Se realizaron dos baciloscopías con un intervalo de dos horas entre ambas. Se calculó el rendimiento diagnóstico adicional definido como el número de baciloscopías positivas solo en la segunda muestra sobre el total de pacientes con baciloscopía positiva en la primera o segunda muestra (11). Para el cálculo del rendimiento diagnóstico adicional solo se incluyó aquellos pacientes que tuvieran ambas baciloscopías.

Las variables cuantitativas se analizaron mediante el cálculo de medias y desviación estándar. Las variables categóricas se presentaron como frecuencias y porcentajes.

La asociación entre la presencia de una baciloscopía positiva y variables cuantitativas, se evaluó mediante la prueba $\mathrm{t}$ de Student; la asociación con variables categóricas fue evaluada mediante la prueba de chicuadrado o prueba exacta de Fisher. Para el análisis multivariado, se incluyeron a aquellas variables que en el análisis bivariado presentan un valor de $p<0,25$. Dichas variables fueron ingresadas en un modelo de regresión logística múltiple. Para evaluar la concordancia entre los resultados de la primera y la segunda baciloscopía se utilizó la prueba de concordancia Kappa.

Se consideró significativo un valor de $p<0,05$. Todos los cálculos fueron realizados utilizando el programa estadístico STATA v.10 (Stata Corp. College Station, Texas 2009).

Se realizaron entrevistas individuales y voluntarias, utilizando una ficha de recolección de datos y un consentimiento informado. El estudio fue aprobado por el Comité de Ética de la Universidad Peruana Cayetano 
Heredia, el Comité de Ética del Hospital Nacional Cayetano Heredia y el Comité de Ética del Hospital Nacional Hipólito Unanue.

\section{HALLAZGOS}

Se evaluaron 824 pacientes, de los cuales 270 cumplieron los criterios de inclusión; 150 correspondientes al $\mathrm{HNCH}$ y 120 correspondientes al HNHU. Cincuenta pacientes $(18,5 \%)$ presentaron al menos una baciloscopía positiva. La frecuencia de baciloscopías positivas fue similar en ambos hospitales: $19,3 \%$ en el $\mathrm{HNCH}$ y $17,5 \%$ en el HNHU $(p=0,70)$.

La edad promedio que se obtuvo de los pacientes incluidos fue de 46,9 \pm 18 ,8 años, siendo la menor edad de 15 años y la mayor de 94 años. En cuanto al sexo se encontraron $194(71,9 \%)$ hombres y $76(28,2 \%)$ mujeres. (Tabla 1).

Con relación a la calidad de la muestra, se puede evidenciar que la proporción de muestras positivas entre las muestras salivales, hemoptoicas y purulentas fue del 5,$8 ; 9,2$ y $23,6 \%$ respectivamente, siendo la diferencia

Tabla 1. Características sociodemográficas y clínicas en pacientes con síntomas respiratorios atendidos en dos servicios de emergencia. Lima, 2011

\begin{tabular}{|c|c|c|c|c|c|}
\hline \multirow{2}{*}{\begin{tabular}{l}
\multicolumn{1}{c}{ Variable } \\
Variables \\
sociodemográficas
\end{tabular}} & \multicolumn{2}{|c|}{$\begin{array}{c}\text { Baciloscopía } \\
\text { positiva } \\
(n=50)\end{array}$} & \multicolumn{2}{|c|}{$\begin{array}{c}\text { Baciloscopía } \\
\text { negativa } \\
(n=220)\end{array}$} & \multirow[t]{2}{*}{$\begin{array}{l}\text { Valor } \\
\text { de } p\end{array}$} \\
\hline & $\mathbf{n}$ & (\%) & $\mathbf{n}$ & $(\%)$ & \\
\hline Sexo masculino & 31 & $(62)$ & 118 & $(53,6)$ & 0,28 \\
\hline Edad en años* & 40,4 & (20) & 60,5 & $(18,3)$ & $<0,01$ \\
\hline Vivir en hacinamiento & 12 & (24) & 56 & $(25,4)$ & 0,83 \\
\hline Contacto tuberculoso & 26 & (52) & 74 & $(33,6)$ & 0,02 \\
\hline \multicolumn{6}{|l|}{ Antecedentes } \\
\hline Tuberculosis previa & 20 & $(40)$ & 100 & $(45,5)$ & 0,47 \\
\hline Asma & 2 & (4) & 43 & $(19,5)$ & $<0,01$ \\
\hline Insuficiencia renal & 1 & (2) & 6 & $(2,7)$ & 1,00 \\
\hline Diabetes & 5 & (10) & 19 & $(8,6)$ & 0,71 \\
\hline Cáncer & 0 & (0) & 5 & $(2,3)$ & 0,59 \\
\hline Infección por VIH & 4 & (8) & 15 & $(6,8)$ & 0,76 \\
\hline Tabaco & 12 & (24) & 46 & $(20,9)$ & 0,63 \\
\hline Alcohol & 14 & (28) & 44 & $(20)$ & 0,21 \\
\hline Drogas & 5 & (10) & 14 & $(6,4)$ & 0,36 \\
\hline Corticoides & 4 & (8) & 2 & $(0,9)$ & 0,01 \\
\hline $\begin{array}{l}\text { Hospitalizaciones } \\
\text { previas }\end{array}$ & 9 & (18) & 67 & $(30,5)$ & 0,07 \\
\hline \multicolumn{6}{|l|}{ Síntomas principales } \\
\hline Tos & 48 & (96) & 215 & $(97,7)$ & 0,49 \\
\hline Expectoración & 44 & (88) & 197 & $(89,5)$ & 0,75 \\
\hline Fiebre & 28 & (56) & 103 & $(46,8)$ & 0,24 \\
\hline Hemoptisis & 12 & (24) & 70 & $(31,8)$ & 0,28 \\
\hline Disminución de peso & 38 & (76) & 116 & $(52,7)$ & $<0,01$ \\
\hline Sudoración & 22 & (44) & 95 & $(43,1)$ & 0,92 \\
\hline Dolor torácico & 34 & (68) & 165 & (75) & 0,31 \\
\hline Disnea & 43 & (86) & 191 & $(86,8)$ & 0,88 \\
\hline
\end{tabular}

Tabla 2. Evaluación del tipo de muestra de esputo, obtenida en los servicios de emergencia de dos hospitales de Lima, Perú 2011.

\begin{tabular}{lcccc}
\hline $\begin{array}{c}\text { Primera } \\
\text { Muestra }\end{array}$ & \multicolumn{4}{c}{ Segunda Muestra } \\
\hline Salival & Salival & Hemoptoica & Purulenta & TOTAL \\
Hemoptoica & 36 & 1 & 60 & 107 \\
Purulenta & 14 & 26 & 5 & 34 \\
TOTAL & 63 & 30 & 100 & 117 \\
\hline
\end{tabular}

estadística $(p<0,01)$. El $41,5 \%$ de pacientes tuvieron una muestra salival en la primera ocasión, significativamente más que el $24,4 \%$ que tuvieron una segunda muestra salival $(p<0,01)$ (Tabla 2).

Las variables sociodemográficas y clínicas asociadas con la presencia de baciloscopías positivas en el análisis bivariado fueron: edad, sexo, uso de corticoides, contacto tuberculoso, y pérdida de peso (Tabla 1).

En el análisis multivariado las variables con asociación significativa con baciloscopía positiva fueron: la pérdida de peso y el tipo de muestra.

De los 270 pacientes incluidos, a 12 no se les realizó una segunda baciloscopía; de estos, cinco tuvieron la primera baciloscopía negativa. En 258 pacientes en quienes se pudo realizar la segunda baciloscopía dentro de las cuatro horas de realizada la primera, 43 (15,9\% IC 95\% 12,3 -21,8\%) tuvieron alguna o ambas positivas (Tabla 3); 34 pacientes tuvieron un resultado positivo en la primera baciloscopía, y se obtuvo nueve baciloscopías positivas adicionales en la segunda muestra, dando así un rendimiento diagnóstico adicional del 20,9\%; (IC95\% $10,0-36,0 \%)$.

El rendimiento diagnóstico adicional fue de $3 / 23$ $(13,0 \%)$ en el $\mathrm{HNCH}$ y $6 / 20(30,0 \%)$ en el $\mathrm{HNHU}$, sin diferencia significativa entre ambos centros $(p=0,17)$. La concordancia de resultados entre la primera y segunda baciloscopía fue de 95,7\%. (Kappa 0,83; p <0,001 y un porcentaje de acuerdo de $96,1 \%$. La diferencia de

Tabla 3. Evaluación de la segunda baciloscopía realizada el mismo día en pacientes con síntomas respiratorios atendidos en dos servicios de emergencia. Lima, 2011.

\begin{tabular}{lccc}
\hline $\begin{array}{l}\text { Primera } \\
\text { baciloscopía }\end{array}$ & \multicolumn{3}{c}{ Segunda baciloscopía } \\
\hline & Positiva & Negativa & TOTAL \\
\hline Positiva & 33 & 1 & 34 \\
Negativa & 9 & 215 & 224 \\
\hline TOTAL & 42 & 216 & 258 \\
\hline
\end{tabular}


proporción de baciloscopías positivas fue 13,2 en la primera frente a 16,3 en la segunda, ( $p=0,02$; test de McNemar).

\section{DISCUSIÓN}

Nuestro estudio evidenció la utilidad de obtener una segunda muestra de esputo el mismo día, la cual brindó un rendimiento diagnóstico adicional del 20,9\%. Ello concuerda con otros estudios $(7,8,12,13)$ por lo que consideramos que puede ser importante para reducir el tiempo de espera; permitiendo un tratamiento precoz y el aislamiento del paciente, en caso de ser necesario.

La utilidad de la baciloscopía del mismo día para pacientes ambulatorios ha sido recientemente evaluada en un estudio multicéntrico realizado en 6466 pacientes (1526 con cultivo positivo) donde se obtuvo una sensibilidad y especificidad del 70,2 y $96,9 \%$ frente a 65,9 y $97,6 \%$ en el caso de la baciloscopía convencional, sin diferencia significativas entre ambas estrategias; sin embargo, un mayor número de pacientes cumplieron con la entrega de dos muestras de baciloscopía en el esquema de un día ${ }^{(13)}$.

Consideramos que nuestros resultados confirman la utilidad de realizar la baciloscopía del mismo día en los servicios de emergencia de nuestro país y que, de implementarse en estos servicios, puede tener un importante impacto para evitar la propagación intrahospitalaria de tuberculosis, la cual es elevada entre el personal de salud (1). En base a esto, la estrategia diagnóstica evaluada permitiría iniciar el tratamiento antituberculoso en el mismo día, lo que contribuiría a la reducción de costos y permitiría reducir la pérdida de pacientes en el seguimiento diagnóstico ${ }^{(6)}$.

Este estudio es aplicable a circunstancias cotidianas, debido a que la mayoría de los centros de atención primaria cuentan con la prueba de Ziehl Neelsen (10) dado que se trata de una prueba sencilla, eficiente y de bajo costo ${ }^{(9)}$. Nuestro estudio evidenció una alta frecuencia de baciloscopías positivas en pacientes que acudieron por síntomas respiratorios a emergencia en ambos hospitales (18,5\%), hecho evidenciado previamente ${ }^{(5)}$ y que consideramos obliga a definir estrategias concretas para afrontar el problema de la tuberculosis en la emergencia como un reto emergente en Salud Pública. Pese a ello, las recomendaciones de la norma técnica nacional de tuberculosis ${ }^{(10)}$ así como las guías de la OPS ${ }^{(9)}$ no incluyen recomendaciones específicas sobre la búsqueda de casos de tuberculosis en emergencia, situación que en base a nuestros resultados consideramos debe abordarse de inmediato a nivel del Ministerio de Salud.

Entre las variables estudiadas, la disminución de peso y el tipo de muestra se asociaron con una baciloscopía positiva. Ello refleja la limitación de los hallazgos clínicos en el diagnóstico de tuberculosis, y refleja la necesidad de contar de manera rutinaria con baciloscopías rápidas en todos los pacientes con síntomas respiratorios que acuden a la emergencia.

Es importante tener en cuenta una adecuada orientación al paciente para obtener una buena muestra de esputo puesto que existe una mayor probabilidad de ser positiva que una muestra salival $(p<0,01)$. Sin embargo, no deben descartarse las muestras salivales, ya que tienen una positividad del $5,8 \%$.

Dentro de las limitaciones del estudio cabe recalcar el no haber realizado cultivos. Incluso así, no creemos que ello invalide estos resultados debido a que el objetivo se basó en la búsqueda de pacientes bacilíferos quienes son los de mayor riesgo en lugares hacinados. Sin embargo, consideramos que el realizar el cultivo pudo haber incrementado la detección de casos de tuberculosis en emergencia, lo que significaría que la frecuencia de tuberculosis posiblemente sea aun mayor. Otra limitación fue el no haber comparado los resultados de las baciloscopías del mismo día con la estrategia convencional, o realizar un estudio de no inferioridad; sin embargo, la literatura revisada demuestra que ambas estrategias son similares en cuanto a sensibilidad y especificidad ${ }^{(7,8,13)}$. De hecho, las últimas recomendaciones de la OMS sugieren que los países que siguen utilizando el modelo estándar para la detección de casos deben considerar un cambio gradual hacia el mismo día del diagnóstico ${ }^{(6)}$.

Por otro lado, cabe recalcar que identificar precozmente a aquellos pacientes con criterios de aislamiento no tendrá un impacto real en reducir la transmisión nosocomial de tuberculosis de no existir la disponibilidad de ambientes físicos de aislamiento; esto implicaría, a su vez, implementar cambios en cuanto a infraestructura adecuada de la unidad de emergencia; capacitación del personal de salud para la toma de muestra; conexión rápida y fluida con el laboratorio; control de calidad de baciloscopías; y organización para la implementación del tratamiento el mismo día lo cual representa un problema para la mayoría de los servicios de emergencia de los hospitales del Perú.

En conclusión, la realización de una segunda baciloscopía el mismo día incrementa el rendimiento diagnóstico para la identificación de pacientes bacilífieros 
entre aquellos que acuden con síntomas respiratorios al servicio de emergencia. Ello resulta especialmente relevante considerando la alta frecuencia observada de casos de tuberculosis en los dos hospitales evaluados. Por ello, sugerimos que la prueba de baciloscopía debe ser incluida como prueba de rutina en los servicios de emergencia de los hospitales del Ministerio de Salud de Lima Metropolitana. Debe considerarse la realización de una segunda baciloscopía durante el mismo día de ingreso al servicio de emergencia pues esto ayudaría a acelerar el diagnóstico y tratamiento de esta enfermedad.

\section{Contribuciones de autoría}

Todos los autores participaron en la concepción del estudio. GT y MU participaron en el reclutamiento de pacientes y colección de los datos del estudio. JA y GT participaron en el análisis de las muestras bacteriológicas. AS, GT y MU participaron en el análisis de los datos y elaboración del manuscrito inicial. Todos los autores revisaron críticamente el manuscrito y autorizaron la versión final.

\section{Fuentes de financiamiento}

Autofinanciado.

\section{Conflictos de interés}

Los autores declaran no tener ningún conflicto de interés.

\section{REFERENCIAS BIBLIOGRÁFICAS}

1. Accinelli R, Noda J, Bravo E, Galloso M, López L, Agapito $J$, et al. Enfermedad tuberculosa entre trabajadores de salud. Acta Méd Peruana. 2009;26(1):35-47.

2. Menzies D, Fanning A, Yuan L, FitzGerald J. Hospital ventilation and risk for tuberculous infection in canadian health care workers. Canadian Collaborative Group in Nosocomial Transmission of TB. Ann Intern Med. 2000;133(10):779-89.

3. Díaz AO, Dueñas D, Lazo MA, Borroto S, González E. Tuberculosis en trabajadores de salud del Hospital Psiquiátrico de La Habana, 1997-2003. Rev Panam Infectol. $2005 ; 7(3): 22-6$
4. Danilla M, Gave J, Martínez-Merizalde N. Tuberculosis ocupacional en un Hospital General de Lima, Perú. Revista de la Sociedad Peruana de Neumología. 2005;49(2):101-5.

5. Solari L, Acuna-Villaorduna C, Soto A, Agapito J, Perez F, Samalvides F, et al. A clinical prediction rule for pulmonary tuberculosis in emergency departments. Int J Tuberc Lung Dis. 2008;12(6):619-24.

6. World Health Organization. Same-day diagnosis of tuberculosis by microscopy. Policy statement. Geneva: WHO; 2011.

7. Cambanis A, Yassin MA, Ramsay A, Squire SB, Arbide I, Cuevas LE. A one-day method for the diagnosis of pulmonary tuberculosis in rural Ethiopia. Int J Tuberc Lung Dis. 2006;10(2):230-2.

8. Rawat J, Biswas D, Sindhwani G, Masih V. An alternative 1-day smear microscopy protocol for the diagnosis of pulmonary tuberculosis. Respirology. 2010;15(7):1127-30.

9. Organización Panamericana de la Salud. Manual para el diagnóstico bacteriológico de la tuberculosis: Normas y guía técnica - Parte 1 Baciloscopia. Argentina: OPS; 2008.

10. Ministerio de Salud (MINSA). Norma técnica de salud para el control de la tuberculosis [Internet]. Lima: MINSA; 2006.

11. Mase SR, Ramsay A, Ng V, Henry M, Hopewell PC, Cunningham $\mathrm{J}$, et al. Yield of serial sputum specimen examinations in the diagnosis of pulmonary tuberculosis: a systematic review. Int J Tuberc Lung Dis. 2007;11(5):485-95.

12. Hirao S, Yassin MA, Khamofu HG, Lawson L, Cambanis A, Ramsay A, et al. Same-day smears in the diagnosis of tuberculosis. Trop Med Int Health. 2007;12(12):1459--63.

13. Cuevas LE, Yassin MA, AI-Sonboli N, Lawson L, Arbide I, Al-Aghbari N, et al. A multi-country non-inferiority cluster randomized trial of frontloaded smear microscopy for the diagnosis of pulmonary tuberculosis. PLoS Med. 2011;8(7): e1000443. Epub $2011 \mathrm{Jul} 12$.

Correspondencia: Gina Paola Tello Villena

Dirección: Asoc. Vivienda El Roble Mz E Lt 20. Lima 6, Perú. Teléfono: (511) 5474102

Correo electrónico: paolatv18@gmail.com

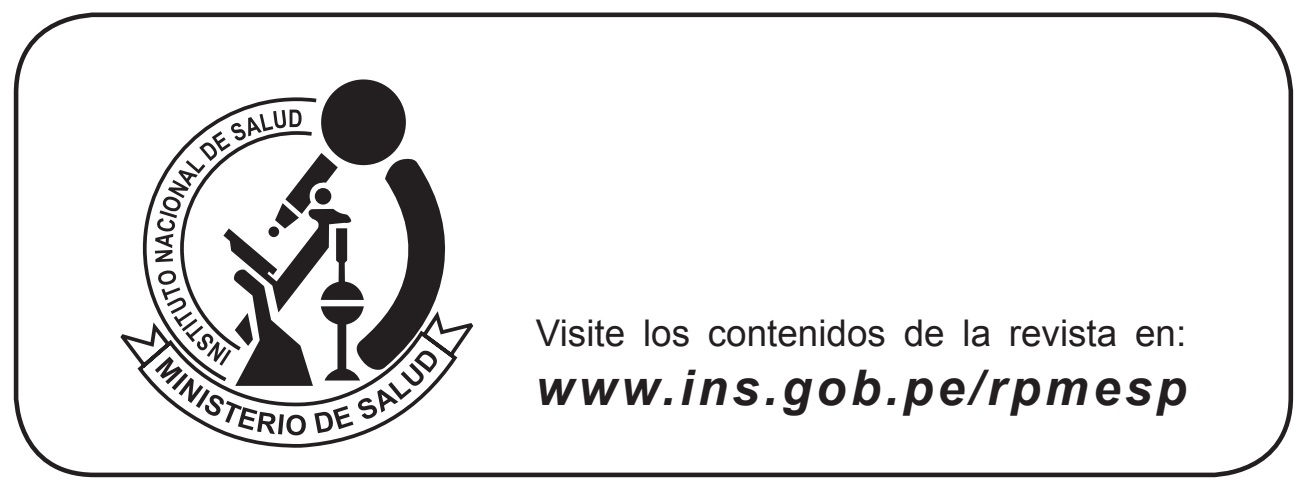

